\title{
AFM and NanoSIMS analyses of Vaccinia virions
}

Peter K. Weber ${ }^{1}$, Sean D. Gates ${ }^{1,2}$, Richard C. Condit ${ }^{3}$, Nissin Moussatche ${ }^{3}$ and Alexander J. Malkin $^{1}$

1. Physical and Life Sciences Directorate, Lawrence Livermore National Laboratory, Livermore, California, USA.

2. Department of Mechanical Engineering, Stanford University, Stanford, California, USA.

3. Department of Molecular Genetics \& Microbiology, University of Florida, Gainesville, Florida, USA.

Characterization of virus virion structure remains a topic of interest in virology. While electron microscopy and X-ray diffraction have provided a wealth of structural data on virions, questions remain regarding the structure and assembly of Pox viruses [1]. Here we explore the potential for high-resolution secondary ion mass spectrometry (SIMS) to be applied to the analysis of the virion structure. This work is technically of interest in the context of a mass spectrometry imaging session because the size of virions pushes the limits of resolution and sensitivity of imaging mass spectrometry. In our work, we are studying Vaccinia, a member of the Pox virus group that is used as the live vaccine for the small pox virus. Like other Pox viruses, the Vaccinia virion is relatively large for a virus $(\sim 350 \times 300 \times 260 \mathrm{~nm}$ hydrated, and $\sim 330 \times 260 \times$ $125 \mathrm{~nm}$ dehydrated), making it an easier virus test subject for high-resolution SIMS. Our intention is to use dynamic SIMS with a monatomic primary ion beam, and therefore we are not seeking to detect molecules directly but rather intend to use isotopic (e.g., $\left.{ }^{15} \mathrm{~N}\right)$ tags to map the distribution of labeled molecules within the virion.

Vaccinia virions in water were dispersed on single-crystal silicon wafers and air-dried for analysis. Samples were first imaged with atomic force microscopy (AFM) to provide virion dimensions and quality control, particularly to control for debris on the virions. For highresolution SIMS, we used a Cameca NanoSIMS 50 with nominal lateral resolution of the $\mathrm{Cs}^{+}$ primary ion beam of $50 \mathrm{~nm}$. The $\mathrm{Cs}^{+}$beam was rastered over the sample to generate secondary ions, which were collected simultaneously on discrete electron multipliers to and used to generate ion-specific digital images of the sample. For this work, up to five species were collected simultaneously. Because the lateral resolution of the primary ion beam was at the scale of the virions, we used an imaging depth profile approach, which has the potential to provide higher spatial resolution in depth than could be achieved laterally [2]. This approach also avoided the need for sectioning. The challenge with this approach for the Vaccinia virions was that a substantial portion of the virion was consumed by sputtering before equilibrium could be achieved, and ion count rates constantly evolved during the course of analysis.

Co-registration between AFM and SIMS was achieved by imaging. The NanoSIMS secondary electron ( $\left.\mathrm{e}^{-}\right)$and ion imaging were able to resolve and image individual virions for positive identification of areas imaged by AFM (Fig. 1). Depth profiles for individual virions were extracted from serial NanoSIMS ion images for individual virions (Fig. 2). Our tests indicated that the $\mathrm{CN}^{-}$species had the highest useful yield of the C-bearing species for iridium-coated virions, and the $\mathrm{C}_{2}{ }^{-}$species had the highest useful yield of the $\mathrm{C}$-bearing species for uncoated virions. We also noted that yield of $\mathrm{CN}^{-}$at the virion surface was enhanced by iridium coating. 
Total ions collected for these species were 100,000 to 1,500,000. Based on these results, we anticipated that sufficient ion counts can be collected from isotopic labeling with ${ }^{13} \mathrm{C}$ and ${ }^{15} \mathrm{~N}$ can provide information on the location of the labeled.

To test our ability to determine the location of specific molecules within the virion, we labeled Vaccinia virions with ${ }^{15} \mathrm{~N}$-thymidine. Initial SIMS results showed that individual virions were labeled to the expected level of ${ }^{15} \mathrm{~N}$ enrichment. However the distribution of ${ }^{15} \mathrm{~N}$ within the virion was skewed relative to expectations. Follow up studies suggest that the rate of sputtering is changing rapidly while sputtering equilibrium is approached. We are currently developing a model of sputtering rate for use with our isotopic depth profiles. The latest results will be presented.

References:

[1] RC Condit, N Moussatche, and P Traktman, Advances in Virus Research 66 (2006), pp. 31 124.

[2] S Ghosal, SJ Fallon, TJ Leighton, KD Wheeler, MJ Kristo, ID Hutcheon, and PK Weber, Analytical Chemistry 80 (2008) pp. 5986-5992.

[3] This work was funded by Lawrence Livermore National Laboratory (LLNL) Laboratory Directed Research and Development and performed under the auspices of the U.S. Department of Energy by LLNL under Contract DE-AC52-07NA27344.
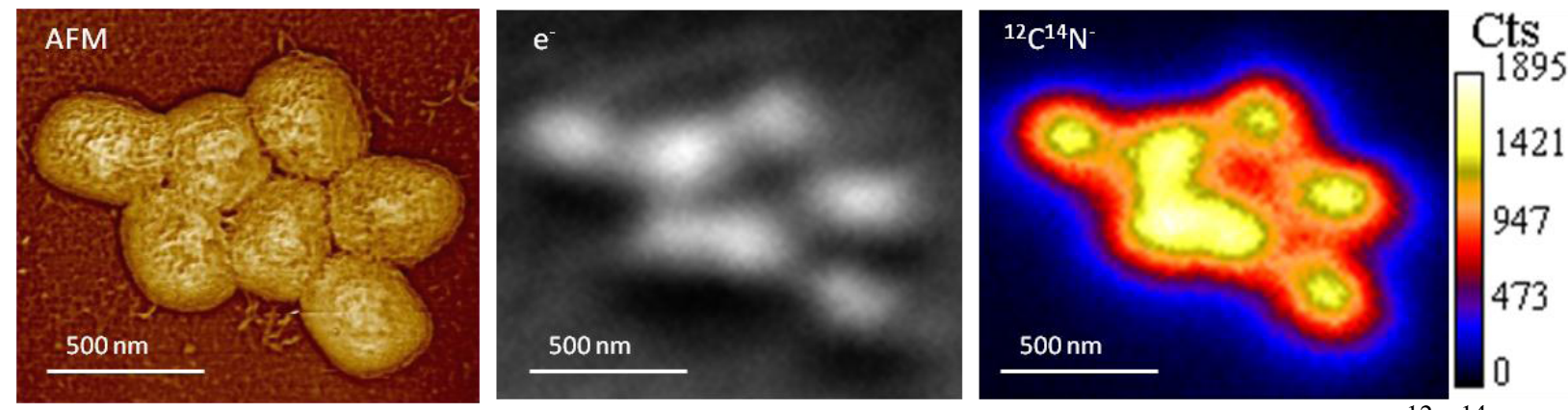

Figure 1. Correlated AFM and depth averaged NanoSIMS secondary electron $\left(\mathrm{e}^{-}\right)$and ${ }^{12} \mathrm{C}^{14} \mathrm{~N}^{-}$ images of vaccinia virions. The virions are consumed in a series of analysis scans.

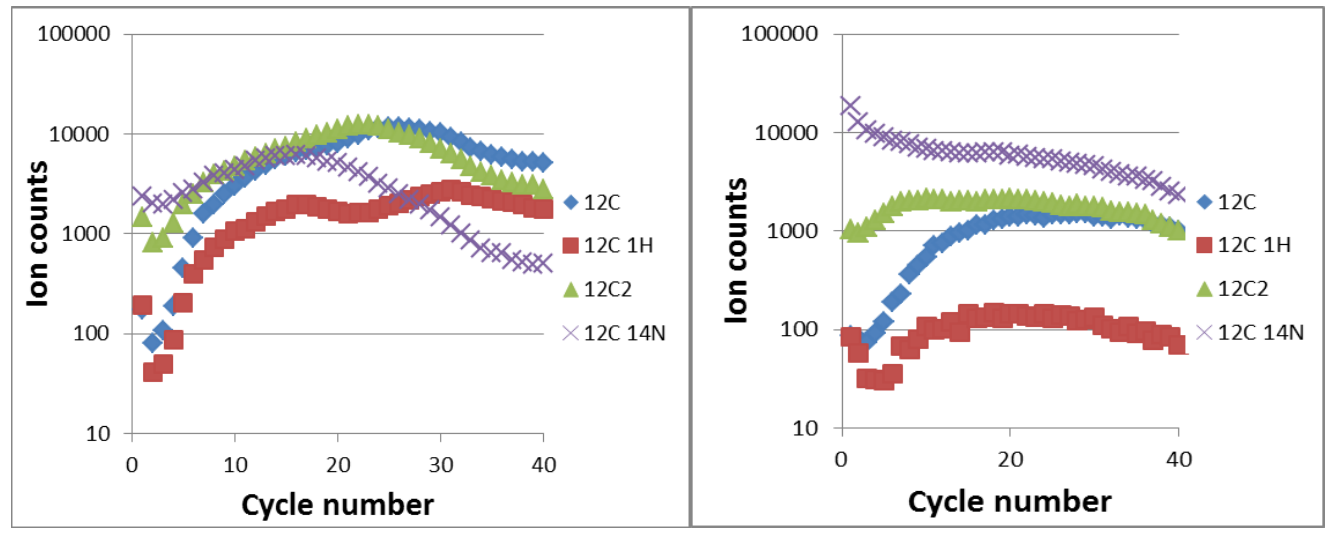

Figure 2. Depth profile of single virionsuncoated (left) and iridium coated (right) showing counts per cycle for four carbonbearing species. 